\title{
Agroecología o Agricultura más que humana?: La coordinación con las plantas como técnica agrícola
}

Agroecology or Agriculture more than human?: Coordination with plants as an agricultural technique

\section{Gabriela Schiavoni}

\section{(2) OpenEdition}

\section{Journals}

Edición electrónica

URL: https://journals.openedition.org/aa/9399

DOI: 10.4000/aa.9399

ISSN: 2357-738X

Editor

Programa de Pós-Graduação em Antropologia Social (UnB)

Edición impresa

Paginación: 150-169

ISSN: 0102-4302

\section{Referencia electrónica}

Gabriela Schiavoni, «Agroecología o Agricultura más que humana?: La coordinación con las plantas como técnica agrícola», Anuário Antropológico [En línea], v. 47 n.1 | 2022, Publicado el 31 enero 2021, consultado el 22 julio 2022. URL: http://journals.openedition.org/aa/9399; DOI: https://doi.org/ 10.4000/aa.9399

\section{(c) (i) (9)}

Creative Commons - Atribución-NoComercial-SinDerivadas 4.0 Internacional - CC BY-NC-ND 4.0 https://creativecommons.org/licenses/by-nc-nd/4.0/ 


\section{Anuário Antropológico}

v.47 n.1 | 2022

2022/v.47 n.1

Agroecología o Agricultura más que humana?: La coordinación con las plantas como técnica agrícola

Agroecology or Agriculture more than human?: Coordination with plants as an agricultural technique

\section{Gabriela Schiavoni}

\section{OpenEdition \\ Journals}

\section{Edição electrônica}

URL: http://journals.openedition.org/aa/9399

DOI: $10.4000 / a a .9399$

ISSN: $2357-738 X$

\section{Editora}

Programa de Pós-Graduação em Antropologia Social (UnB)

\section{Referência eletrônica}

Gabriela Schiavoni, «Agroecología o Agricultura más que humana?: La coordinación con las plantas como técnica agrícola», Anuário Antropológico [Online], v.47 n.1| 2022. URL: http://journals. openedition.org/aa/9399; DOI: https://doi.org/10.4000/aa.9399

Anuário Antropológico is licensed under a Creative Commons. Atribuição-SemDerivações-SemDerivados CC BY-NC-ND 


\title{
Agroecología o Agricultura más que humana?: La coordinación con las plantas como técnica agrícola
}

\author{
Agroecology or Agriculture more than human?: Coordination with plants as an \\ agricultural technique
}

DOI: https://doi.org/10.4000/aa.9399

\section{Gabriela Schiavoni}

Universidad Nacional de Misiones, Posadas (Misiones) - Argentina

Antropóloga, investigadora del Conicet y docente del Programa de Posgrado en Antropología Social de la cológica, indígena y colona y sobre las técnicas aplicadas al mejoramiento vegetal y a la producción de alimentos.

Focalizando en la agricultura de roza y quema, propia de los grupos indígenas y campesinos de las regiones tropicales y subtropicales del globo, este artículo describe diversas formas del vínculo entre los hombres y las plantas. La noción de agricultura más que humana comprende los aspectos no intencionales de la domesticación, haciendo recaer la operación técnica en un organismo mixto humano-vegetal. La reflexión está arraigada en la provincia de Misiones (nordeste de la Argentina) y su región circundante (Paraguay y sur de Brasil). Recuperando elementos de la agricultura guaraní, tales como el consorciamiento de cultivos, establecemos un puente con la agroecología actual.
Focusing on swidden agriculture, characteristic of the indigenous and peasant groups of the tropical and subtropical regions of the globe, this article describes various forms of the link between men and plants. The notion of agriculture more than human includes the unintended aspects of domestication, making the technical operation fall on a mixed human-plant organism. The reflection is rooted in the province of Misiones (northeast of Argentina) and its surrounding region (Paraguay and southern Brazil). Recovering elements of Guaraní agriculture, such as crop consortia, we establish a bridge with contemporary agroecology. 


\section{Introducción}

La imitación de la naturaleza es el hilo que enlaza las distintas versiones de la agroecología. Formulada a principios del siglo XX, en términos filosóficos antes que técnicos (Besson 2009), el proceder presenta analogías con los saberes premodernos de los siglos XV y XVII. Supone leer la prosa del mundo en la llanura uniforme en la que las palabras aún no se han separado de las cosas (Foucault 1966), apelando a las semejanzas engendradas por conveniencia, emulación, analogía y simpatía.

Consideradas en esta clave, las entidades se afectan unas a otras y las tramas asociativas se establecen por contigüidad, siguiendo las marcas presentes en la superficie, sin el recurso a un principio explicativo externo (Racine 1989). En tanto método indirecto y oblicuo, la analogía franquea el acceso a algunos secretos de la naturaleza (Bemakhlouf 1999), configurando "un modo de identificación que descompone los existentes en una multiplicidad de esencias, formas y sustancias separadas por pequeñas distancias" (Descola 2012, 301). El objetivo de este artículo es retomar dichas lecturas con miras a poner en evidencia las interacciones vegetales en los procesos de domesticación, concibiendo la agricultura como una operación mixta, en la que los humanos no son los demiurgos transformadores.

Objetivadas inicialmente por la medicina, las afinidades entre las plantas consideradas simplicia o remedios provistos por la naturaleza - constituyeron la materia médica hasta fines del siglo XVIII. Así, Descola extrae de la historia de la medicina dos ejemplos a partir de los cuáles desarrolla la ontología analogista: la teoría de las signaturas y el esquema frío-calor.

La simpatía-antipatía entre especies, mencionada en la materia médica de Dioscórides, y también en los libros de agricultura de la modernidad temprana (Mafferra 2019), es inductora de efectos, tanto terapéuticos como ecológicos. De este modo, la hostilidad entre el helecho y la caña hace que el jugo del helecho cure las heridas causadas por las flechas hechas de caña, y "la prueba de esto es que donde hay mucha caña y muchos helechos rodeando, el helecho se desvanece” (Dióscorides en Willis 2007, 24-5).

La sociabilidad entre especies alcanza también al ámbito culinario: la enemistad entre las coles y la vid hace "que si guisais coles en una olla y echais vino en ella, nunca se coserán” (Agustín 1722, 62). A su vez, la similitud de forma entre partes de la planta y órganos del cuerpo humano es un indicio de su acción curativa, de acuerdo a la doctrina de las signaturas.

El estudio de las propiedades observables y de las interacciones entre vegetales será eclipsado en las primeras décadas del siglo XIX por los avances de la química, que injertará un código experto en el diálogo con la naturaleza. Esto culminará con los trabajos de Julius Liebig sobre el papel determinante de los elementos minerales mayores en la agricultura.

Aún así, el fenómeno de la influencia entre plantas se mantendrá como un tema de estudio de la fisiología vegetal hasta principios del siglo XX. De Candolle, en el siglo XIX, explicará el efecto de la rotación de cultivos atendiendo a la 
secreción de las raíces. En 1937, Hans Molisch acuñará el término alelopatía para designar los efectos de la interacción entre dos vegetales.

En la modernidad tardía, el retorno de las lecturas premodernas es detectable en diversas perspectivas, entre ellas la agroecología, como reacción frente al reduccionismo químico del naturalismo. El reconocimiento del carácter premoderno del pensamiento de los fundadores (Howard, Steiner, Rusch, y Fukuoka) no va en desmedro del enfoque, sino que aumenta su inteligibilidad (Besson 2009).

En aras de dar cuerpo a esta agricultura más que humana, centro mi atención en la interacción vegetal, una de cuyas expresiones es la asociación del poroto con el maíz, considerada una herencia prehispánica que se extiende desde el Río de la Plata hasta Mesoamérica (Garavaglia en Boidin 2005).

La complementariedad entre vegetales, promovida por la agroecología en la segunda mitad del siglo XX en la región que estudio - la provincia de Misiones, en el noreste subtropical de Argentina -, abrió la posibilidad de una mediación técnica entre los humanos y las plantas basada en la imitación de la naturaleza. Las formas que describiremos aquí han sido engendradas en la fricción entre lecturas que obedecen a la naturaleza, lecturas que tratan de dominarla, y otras que intentan reproducirla. Asentada en una realidad enmarañada, la labor compartida de vegetales y humanos coexiste con el proyecto monoespecie de la modernidad, en el que proliferan los vivientes construidos como objetos técnicos abstractos.

El punto de partida de mi argumentación es la agricultura selvática, propia de los grupos indígenas y campesinos de las regiones tropicales y subtropicales del globo. Este estilo de agricultura ha sido denominado de varias formas: schifting cultivation, nómade, swidden, de roza y quema, con barbecho largo (Conklin 1954, Geertz 1970, y Barrau 1967), y, en el caso de Misiones como agricultura, de coivara. En virtud de su asociación frecuente con el cultivo de tubérculos, fue caracterizada también como horticultura y vegecultura (Sigaut 1982, Gourou 1949), constituyendo el referente concreto del esquema de amistad respetuosa entre los humanos y las plantas, propuesto por Haudricourt (1962). Bajo el rótulo de botanista, Sigaut (1982) la distingue de la agricultura mecanicista, señalando que en este caso la tendencia técnica es hacia el mejoramiento del material vegetal. La ausencia del arado, y a veces de la azada, es considerada por Padoch (2018) como índice de agroforestería. También Ellen (2012) enmarca la agricultura de swidden en las prácticas de restauración de la selva y la biodiversidad.

Este repertorio de variaciones retiene como fundamento la existencia de un vínculo no selectivo con el ambiente, en el que el formato productivo retoma como información las singularidades de los sistemas vivientes, en fricción con las simplificaciones ecológicas de la plantation. La no escalabilidad del ensamblaje permite que ambos mundos coexistan, de manera que la agroecología emerge como producto de contradicciones y su dinamismo radica en la fricción.

Mi razonamiento abreva en distintas fuentes y está arraigado en la provincia de Misiones, un espacio tardíamente incorporado a la nación Argentina, cuyo poblamiento moderno, de origen inmigratorio y proveniente del centro y este de Europa, se desarrolló durante la primera mitad del siglo XX, a través de empresas 
colonizadoras en la franja costera del Río Paraná, y mediante la acción del Estado en la zona sur y área central. En forma simultánea y preexistente se desplegaron las dinámicas poblacionales autogestionadas, que comprenden los asentamientos móviles de los grupos mbya guaraní y la ocupación espontánea llevada a cabo por pequeños agricultores no indígenas en las tierras fiscales vacantes del nordeste provincial.

En este contexto, la agroecología se configuró como un devenir de colonos inmigrantes, localizados mayormente en depto. Gral. San Martín, que, ante el agotamiento de los suelos, se orientaron hacia operaciones de imitación de la naturaleza, luego de haber practicado una agricultura convencional (Schiavoni 2020). En efecto, el poblamiento moderno de la provincia se estableció en contraposición a la agricultura montaraz y a las prácticas indígenas de vinculación con la naturaleza, que intento reconstruir aquí utilizando testimonios históricos, tales como el herbario guaraní-jesuítico del siglo XVIII y los escritos del naturalista suizo Bertoni, radicado en la región a fines del siglo XIX. En las tres últimas décadas, el afianzamiento de la agroecología instauró un nuevo diálogo con estos antecedentes, permitiendo una revisión de la experiencia de los colonos, en la que aflora la cooperación inicial con la población nativa. Enlazo estos datos con mi propio trabajo etnográfico, acompañando desde 1990 los procesos de instalación agrícola en el frente pionero (depto. San Pedro, Schiavoni 1995). En virtud del carácter no planificado de este poblamiento, los pequeños productores ocupantes entablan con el ambiente vínculos de familiaridad que, sin embargo, no desembocan en prácticas agroecológicas, ya que su esquema técnico explota la fertilidad inicial de los suelos. ${ }^{1}$

\section{Mediar la naturaleza: la técnica}

En el mundo occidental, la comprensión de la materia vegetal movilizó distintas ontologías. Inicialmente, fueron las propiedades observables, luego se pasó a la identificación de sustancias orgánicas y, finalmente a los compuestos de carbono, sólo accesibles a los expertos (Klein 2005). Estos formatos cambiantes desplazaron el análisis de lo viviente, aproximándolo a la química inorgánica. Liebig, el fundador de la agronomía moderna, es un exponente de este tercer momento que hegemonizó la técnica vegetal, deteniendo el flujo de lo viviente y fijando teóricamente las reacciones al ambiente a través de la creación de una "planta tipo”, de naturaleza ideal.

Fueron dejadas de lado las operaciones divergentes con respecto a este patrón, tales como el tratamiento ensamblado de especies y ambientes, propio de la agricultura campesina. En su estudio del cultivo de papas en Perú, van der Ploeg da cuenta de este formato cuando relata: "Con frecuencia escuché a los agricultores afirmar que tal o cual tubérculo no podía ser un calhuay ya que no podría cultivarse más que en determinados ambientes" (van der Ploeg 2000, 366).

A semejanza de la botánica premoderna, el proceder campesino no aísla individuos sino que experimenta la naturaleza como conexiones, engendrando entidades mixtas que involucran humanos, vegetales y ambientes. Lazos de este tipo
1 Distintas versiones agroecológicas coexisten en Misiones, tales como la biodinámica y la agroforestería, más reciente. La agricultura orgánica fue difundida a través de programas estatales de producción de alimentos sin configurarse como una identidad política. En 2014 se sancionó una ley provincial de fomento a la producción agroecológica. 
también se encuentran en los mitos amerindios relativos al origen de las plantas cultivadas, en los que el carácter salvaje o domesticado de una planta depende del trato verbal que le dispensen los humanos, como es el caso de las enredaderas que crecen en el borde de los sembrados, denominadas camapu o camuri (Lévi Strauss 1971).

A diferencia de lo que ocurre en el mundo industrial, en las sociedades estudiadas por la antropología, la técnica no se comporta como un extranjero con respecto a la cultura. Los fenómenos técnicos constituyen en ellas una acción distribuida, que se procesa en interacción con los materiales y con los demás vivientes.

La noción de cadena operatoria de Leroi-Gourhan (1964) asimila el hacer humano al comportamiento de un organismo, que actúa por sí mismo a través del entrelazamiento observable de los gestos. Ingold (2013) critica el carácter lineal de esta aproximación, enfatizando el proceder rizomático de la interacción entre los humanos y los materiales. Lemonnier (2013), por su parte, insiste en la necesidad de tomar en cuenta los modos en los que actúa la materia, otorgando relevancia a los aspectos físicos en la determinación del significado. Coupaye (2015) busca conciliar el énfasis descriptivo de la tradición francesa con la antropología interpretativita y los devenires técnicos de Ingold, proponiendo como herramienta la noción de transecto, que conecta lo técnico con lo religioso y lo mágico a través de la experiencia del recorrido.

Ferret (2012), a su vez, desarma la noción occidental de domesticación y coloca la atención en la forma de las acciones, cconsiderando no sólo la operación - un sujeto que actúa sobre un objeto -, sino también la virtual delegación del sujeto al objeto para llevar a cabo el cometido (hacer que el otro haga o manipulación). Este carácter mixto o coactivo de la domesticación fue señalado por Haudricourt y Hédin en El hombre y las plantas cultivadas (1943), otorgando un lugar destacado a las ideas del botánico ruso Vavilov y al papel que este le asignara a las plantas involuntariamente domesticadas (malezas, que al ser cosechadas junto con las especies cultivadas, se auto-domestican). Asimismo, la dependencia con respecto a un contexto espaciotemporal y los parecidos dan lugar a las plantas imitadoras, parásitos anuales de los cultivos que escapan a la destrucción del deshierbe, originando variedades que siguen el ritmo de las cultivadas y presentan con ellas notables analogías biológicas. Una de estas es el centeno, cuyas semillas eran cosechadas y resembradas junto con las del trigo tierno. También algunas leguminosas quedan vinculadas espontáneamente al hacer humano porque su tegumento grueso les permite sobrevivir al fuego y permanecen en los campos cultivados. Así: "Al asociar una leguminosa forrajera a un cereal, nuestro agricultor imita una asociación de las sabanas herbáceas” (Haudricourt y Hédin 1943, 89).

Barrau, discípulo de Haudricourt, avanza en esa misma dirección al situar la agricultura selvática en continuidad con la recolección (Barrau 1967, 1975), a partir de sus observaciones al interior de la isla Guadalcanal, donde los bulbillos aéreos, presentes en los tallos trepadores de los ñames silvestres, caen al suelo removido por los recolectores y se encuentran accidentalmente cultivados. 
Descubiertas empíricamente, frecuentemente en las zonas de intersección de lo silvestre con lo cultivado, las compatibilidades inter-especie constituyen el fundamento no-intencional de la domesticación, que involucra así a un organismo técnico compuesto, humano-vegetal. Concebido en términos inter-especie, el trabajo de producir ya no es obra exclusiva de los humanos, considerados «fuera» del mundo natural, planificando y ejecutando racionalmente diseños (van Dooren 2012). Las entidades supraindividuales así engendradas recuperan la mixtura o proceder distintivo de constitución de la unidad ejecutado por las plantas, basado en la proyección recíproca sin pérdida de identidad (Coccia, 2017).

Las operaciones en las que descansa este régimen de coactividad resultan afines a las lecturas premodernas de la naturaleza, ya que retienen la dependencia con respecto al contexto espaciotemporal, así como los parecidos ostensibles. Mediado por los humanos, el compañerismo interespecífico deviene una técnica al ser reproducido o emulado intencionalmente. Las distintas versiones de la agroecología contemporánea enuncian e inscriben compatibilidades de hecho entre las especies, de manera que los objetos técnicos así construidos no tienen su inicio en una imagen mental sino en la experiencia de modos de funcionamiento existentes.

Este formato técnico, a diferencia de la agricultura industrial, no es escalable, ya que resulta sensible a las contingencias, haciendo que el trabajo conjunto con las plantas implique interactuar con una fuerza distribuida. La argumentación que sigue recorre tres pasos, describiendo las operaciones de seguir, imitar y producir naturaleza.

\section{Seguir a la naturaleza: naturalistas y recolectores}

Buscando leer lo que nunca ha sido escrito, naturalistas y recolectores establecen con el ambiente un intercambio complejo. Barrau (1967) afirma que los recolectores son notables naturalistas, en virtud del contacto estrecho que mantienen con el mundo vegetal.

En la región que estudio, los guaraníes "desgraciadamente (...) no dejaron ningún documento escrito (...) pero la ciencia de la raza está escrita (...) en decenas de miles de vocablos técnicos" (Bertoni 1940, 150). La familiaridad y el conocimiento indígena de las plantas se conservan en los herbarios sudamericanos del siglo XVIII, emanados de los recorridos efectuados por los jesuitas junto a los indígenas. La Materia Médica Misionera (1710) del padre Montenegro (1663-1728) recoge esta experiencia, incluyendo las denominaciones guaraníes. ${ }^{2}$

Confluyen en este herbario el proceder de los recolectores-agricultores indígenas y los saberes botánicos premodernos europeos sobre plantas medicinales. Inspirado en Laguna y Mathioli, comentaristas de Dioscórides, el jesuita describe los especímenes de la región según los temperamentos frío/ caliente y húmedo/ seco de la medicina galénica, de cura por los contrarios.

Así, la yerba mate, primera planta del herbario, cuyas propiedades medicinales consisten en "curar heridas recientes, arraigar dientes y muelas que se mueven, por corrimientos de calor y socorrer a los asoleados" es caracterizada en los
2 El otro herbario data de 1725 y fue compuesto en lengua guaraní, por Marcos Villodas. El manuscrito de Montenegro, escrito en castellano, está conservado en dos códices, uno de los cuáles fue reeditado por la Editorial Universitaria de la Universidad Nacional de Misiones en 2007, y es el que utilizamos aquí. El otro, fue publicado por Martín y Valverde en 1995. 
siguientes términos: "Consta de partes calientes en el primer grado, y de secas en el segundo: tiene algunas frías en la superficie” (Montenegro 2007, 92).

Descola (2012) subraya el carácter arbitrario y convencional de estas distinciones, señalando que: "Lo caliente y lo frío (...) actúan (...) de encabezados abstractos y convencionales para subsumir pares de contrarios, no de indicadores empíricos de un estado material” (Descola 2012, 325). En la lectura del jesuita, sin embargo, caliente y frío hacen referencia a propiedades concretas. Así, "las cualidades actuales de calor, humedad. frialdad o sequedad, se disciernen y conocen por el tacto, cuyo principal instrumento es el cuerecito interior de los dedos" (Montenegro 2007, 65-6). La calificación del temperamento incluye también los ambientes en los que crecen los especímenes. De este modo, en la descripción de la ipecacuana (isipó moroti), leemos: "Es el bejuco caliente en el segundo grado, y seco en el tercero, aunque parece consta de partes frías al sabor (...) por la cualidad de la tierra adonde nace no darle el sol de ordinario" (Montenegro 2007, 199).

La conexión con los animales también es movilizada en este formato de caracterización de lo viviente. Así, el Kaápebá o palo de las culebras es descripto como "una pequeña enredadera, que de ordinario se halla a las orillas de los arroyos y ríos”, y lo llaman así en Brasil porque "además de ser parecido a ellas en sus partes, es el más eficaz remedio que ellos usan en las mordeduras de todas las serpientes que arrastran por el suelo" (Montenegro 2007, 142).

Las láminas, consideradas por Martín y Valverde (1995, 44), "lo más sobresaliente que hay en el manuscrito", son solidarias de esta lectura figurativa, anterior al surgimiento de la nomenclatura codificada. Subrayan la ausencia de clasificación, afirmando que: "la cuestión de la sistemática debió ser totalmente secundaria ya que la identificación de plantas se hacía señalando caracteres externos fácilmente perceptibles de visu" (Martín y Valverde 1995, 51). Con respecto a las denominaciones guaraníes que aparecen en el manuscrito, agregan que "son denominaciones vulgares y que, por ser idioma pobre en palabras, designaban con el mismo nombre una familia entera de plantas (Martín y Valverde 1995, 51).

Sin embargo, la economía y el uso repetido de términos de esta nomenclatura será valorizado por el naturalista suizo Bertoni, a fines del siglo XIX, como evidencia de una sistemática basada en el concepto de familia natural, propio de la botánica anterior a Linneo. ${ }^{3}$ Asociada al nombre del botánico Adanson, esta forma de clasificación supone "ver, escuchar y seguir a la naturaleza, arrancándole el secreto de las reglas que han de seguirse en la formación de géneros en cada una de las diferentes familias" (Lagasca 1826).

Al formular la sistemática guaraní, Bertoni retiene el carácter colectivo y deliberativo de esta nomenclatura, establecida mediante asamblea, subrayando la importancia del nombre, que contiene parte del ser que designa, y "un nombre errado o inconveniente le quita al ser algo de su esencia” (Bertoni 1940, 151).

A semejanza de los mitos y los ritos, recolectores y naturalistas asocian entidades por parecidos y atributos perceptibles, preservando "modos de observación y de reflexión (...) a partir de la organización y la explotación especulativas del mundo sensible en términos de sensible (Lévi-Strauss 1962, 25). La vigencia de
3 Bertoni no menciona los herbarios jesuíticos. El ejemplar del manuscrito de Villodas que integra su colección fue adquirido en 1957, por su hijo (Otazú Melgarejo 2014). 
estos criterios es atestiguada por la doctrina de la signatura o similitud que informa la farmacopea guaraní actual (Keller, 2007).

Esta lectura de lo viviente se mantendrá en fricción con las formas de vinculación con las plantas de la agricultura moderna. Así, el valioso esquinanto o kapi’i kachî (Aloysia citrodora) detectado mediante el olfato por el jesuita Montenegro y los indios que lo acompañan (Fleck, 2017) se convertirá para los colonos en el "indomable jahapé” (Imperata brasiliensis, Bertoni 1927, 476), cuya propagación rizomática amenaza los cultivos. Del mismo modo, no será apreciada por los colonos la relación de simpatía que establecen los guaraníes entre las especies sembradas $y$ otras que crecen en el rozado, tales como las achiras [Canna y Thalia paniculatas], denominadas pehuajó y que "no hay que sacar (del cultivo) para que lo sembrado prospere" (Cadogan 2011, 134). ${ }^{4}$

La actividad de cultivar, sin embargo, no entraña siempre un enfrentamiento con lo viviente. Las tradiciones agrícolas que examinaremos a continuación retoman asociaciones existentes y conjugando operaciones voluntarias y espontáneas engendran artefactos humano-vegetales.

\section{El poroto y el maíz: Una agricultura en intersección con lo silvestre}

En el origen del maíz domesticado en Tehuacán (México), Cresswell (1983) señala que también se encuentra el poroto. A su vez, en los mitos amerindios relativos al origen de las plantas cultivadas la única referencia al poroto es a una variedad silvestre que integra el conjunto de vegetales consumidos durante los meses de escasez en la zona del Chaco (Lévi-Strauss 1971, 85). Su condición silvestre se hace patente en la exigencia de hervor previo al consumo con el fin de despojarlo del carácter venenoso o amargo. También tóxico, y por lo tanto silvestre, es el poroto descripto por el jesuita Sánchez Labrador, cuando recorre la región de Paraguay aledaña a Misiones y relata: "Los Infieles chanas (...) tienen en sus bosques una especie de frijoles venenosos, o Cumanda Yohá" (Sánchez Labrador en Fleck y Alliatti 2017, 35-6). La posición fronteriza del poroto, en la intersección de lo silvestre con lo cultivado, había sido reportada por el jesuita Montoya en el siglo XVII, consignando una variedad de la región que recibe la denominación de kokue ryva, porque crece espontáneamente en los espacios que antes fueron chacra (Montoya 2011, 254).

La presencia no intencional del poroto en los campos sembrados con maíz se convertirá en artefactual, constituyendo el germen de objetos técnicos, tales como las prácticas de rotación y consorciamiento de cultivos. La operación que subyace a esta asociación no es el parecido sino la dependencia con respecto al contexto espaciotemporal.

Mezclar y acercar especies vegetales constituye una técnica de la agricultura selvática, practicada en lugares en los que "la naturaleza salvaje es poderosa, donde los animales son constantemente temidos, donde el suelo está mal desbrozado y dado vuelta, hay interés en juntar las semillas en los mejores lugares y enterrarlas inmediatamente" (Leroi-Gourhan 1973, 125-6). Tubérculos y semillas se entierran, haciendo "un agujero con el palo cavador, o con la azada y el agujero se
4 En el relato autobiográfico de un colono de Misiones, leemos: "había yuyos que no existían en otra parte (...) Esas achiras nos dieron mucho trabajo (...) Si no se arrancaban las plantitas chicas, se perdía la batalla" (Müller 1995, 29). 
tapa después con el pie. La siembra al voleo es rara" (Leroi-Gourhan 1973, 125-6).

La siembra apretada de maíz en nuestra región de estudio fue registrada por el jesuita Montoya mediante la expresión: "sembrar el maíz muy junto, o muchos juntos, apeñuscados en montones" (amombeju mbeju avati itỹma, Montoya 2011, 330). También el especialista del gobierno nacional que visita Misiones a principios del siglo XX, describe la siembra conjunta de maíz y poroto, en el marco de la agricultura montaraz con empleo del saracuá, "palo puntiagudo que se va enterrando aquí y allá y en cuyos agujeros se arrojan los granos de maíz y porotos" (Yssouribehere 1904, 99).

La mezcla de especies será interpretada como ahorro de tierra en los contextos campesinos de las tierras bajas sudamericanas, marcados por la exigüidad de los predios. En su etnografía de los campesinos de Pernambuco (nordeste brasilero), Heredia (1979) refiere que, además de la rotación de cultivos, llevan a cabo la práctica de "misturar", cultivando distintos productos en una misma parcela, sembrándolos en hileras separadas o poniendo varios tipos de semillas diferentes en un mismo pozo. La autora detalla la participación de la forma vegetal en esa cooperación, señalando que el maíz sólo se asocia con una variedad trepadora de poroto, ya que es imposible sembrar otros cultivos en el mismo pozo porque el maíz crece rápidamente y produce demasiada sombra lo cual dificulta el crecimiento de otras plantas. La fava (Phaseolus lunatus), por ser una variedad trepadora y más resistente que los otros porotos, se enrolla en el maíz creciendo ambos sin dificultades.

También en el nordeste brasilero, en Sergipe, Woortmann (1997) describe el consorciamiento, de poroto y maíz, codificado en este caso por la calificación frío/calor, con miras a desarrollar vínculos de apoyo entre las plantas y evitar la competencia. Las plantas calientes, como es el caso del maíz, interfieren con las demás, y tienen que ser neutralizadas por plantas frías (el poroto es frío hasta cierto momento del crecimiento).

Este compañerismo entre especies fue observado también en poblaciones guaraníes del sur de Mato Grosso (Brasil) a mediados del siglo XX (Schaden 1974), y entre campesinos actuales de la región oriental del Paraguay (Isla Guazú, depto. Ñembucú, Boidin 2005). Los grados de intimidad oscilan entre la sucesión (sembrar poroto después del maíz), el plantío entre líneas (intercalar poroto con maíz) y la siembra en el mismo hoyo o siembra jopará (mezcla).

Entre los kaiová y los ñandeva del sur de Mato Grosso, Schaden (1974) observa que "para el poroto (kumandá) hay dos épocas de plantío. Una coincide con la del maíz, plantándose el poroto incluso en la misma roza, 'de mistura' para ser cosechado en la primera quincena de noviembre" (Schaden 1974, 39). Boidin (2005) registra este tipo de siembra en el momento de la primavera; consignando la mención del plantío conjunto de poroto rojo y maíz tupí en comunidades guaraníes de la zona del Alto Paraná, si bien el calendario agrícola registrado en dicha zona por el padre Müller a principios del siglo XX no incluye la siembra conjunta, puesto que afirma que plantan el maíz en agosto y el poroto en septiembre. ${ }^{5}$

En Paraguay Bertoni identificó a principios del siglo XX un poroto-maíz ( $k u$ -
5 Menciona el plato djopará (Muller 1989), elaborado guisando poroto y maíz, una preparación frecuente en la dieta de los peones de los obrajes de extracción de madera y yerba de la región. 
mandá avati), que se acostumbra a sembrarlo junto con el maíz tardío (probablemente con la variedad morotí guasú), en plantación asociada (Bertoni 1927, 456). Da cuenta de esta práctica en términos de estrategia de intensificación, mediante la siembra tardía y la realización de dos o tres cosechas anuales de maíz. ${ }^{6}$

También en Misiones, el botánico Martínez Crovetto (1968) consigna la presencia de dicho poroto-avati, plantado junto al maíz pará en los huertos mbya guaraníes de la década de 1960, en los que detecta asimismo la combinación de maíz tupi y poroto soperí (Phaseolus lunatus o poroto manteca). El cultivo consorciado ha sido considerado propio de la agricultura guaraní, haciendo que el rozado adquiera una conformación semejante a la de una huerta (Souza 1987, 99-100). En las aldeas guaraníes actuales de Misiones, hemos observado la siembra conjunta de maíz y poroto, así como la presencia del denominado poroto-maíz. $\mathrm{Y}$, si bien esta agricultura es considerada marginal y su especificidad no es reconocida, ${ }^{7}$ contempla un manejo preciso del tiempo mediante el escalonamiento de maíces con ciclos de duración variable, así como la experimentación con ciertos especímenes, tales como la sandía, denominada chanjáu porque es sembrada el día de San Juan en pequeña cantidad, y de acuerdo al resultado, se la cultivará en septiembre.

La promiscuidad del rozado es mencionada a propósito de grupos mbya guaraní del sur de Brasil (Felipim 2001), entre los que es frecuente la mezcla entre las variedades que conforman el maíz verdadero. Plantadas relativamente próximas e intercaladas con otros cultivos (mandioca, batata, maní, tabaco), "ayudan a asegurar el casamiento entre distintas variedades de maíz" (Felipim 2001, 77-8). También Emperaire y Carneiro da Cunha (Emperaire 2010, 57), describiendo la agricultura tradicional del alto y medio Río Negro, en la amazonia brasilera, observan que el rozado es concebido como un espacio de sociabilidad, cuya forma redonda establece una lógica interna, definiendo un centro que materializa el origen.

Con el fin de neutralizar la influencia derivada de la proximidad, la práctica de la siembra distante es activada a propósito de las plantas que se busca diferenciar. Entre los achuar, Descola (1988) menciona que el maíz se planta solo, porque no es una producción principal. Entre los guaraníes estudiados por Schaden (1974), el maíz ceremonial se planta aparte y demanda condiciones especiales. ${ }^{8}$ También Woortmann (1997) reporta que, en Sergipe, "siempre que se pueda, el maíz blanco se planta en un terreno alejado" (Woortmann 1997, 93). Emperaire y Carneiro da Cunha (2010) refieren, asimismo, que "las mandiocas específicas para el caxiri [bebida fermentada] son plantadas aparte" (Emperaire 2010, 61).

El formato redondo de las rozas, propicio a la interacción vegetal, caracterizó la agricultura montaraz de Misiones anterior a la colonización, y fue descripto por el enviado del gobierno nacional en estos términos: "Siembran (...) pequeñas 'rueditas' (...) término pintoresco (...) con que designa a los pequeños cuadros donde se ven muestras de cultivos indispensables para la subsistencia: maíz, mandioca, batata" (Yssouribehere 1904, 52). Si bien la forma redonda se encuentra actualmente sólo en algunos rozados indígenas, la siembra apretada y el consorciamiento de especies son habituales en los espacios destinados a la producción
6 Señala que fue Rodríguez de Francia, en las primeras décadas del siglo XIX, "quien enseñó a nuestros campesinos que el maíz se podía plantar dos veces en el año" (Bertoni 1927, 120).

7 Encuadradas en la agricultura familiar estatal, las comunidades indígenas desarrollan una producción de huerta con miras a su incorporación a ferias.

8 Sus interlocutores señalan que el maíz ceremonial sólo crece en la capuera (Schaden 1974, 41). 
de autoconsumo en el conjunto de agricultores familiares de la provincia.

Es decir, el consorciamiento y la disposición de los vegetales (proximidad o distancia), así como el modo y las herramientas empleadas para enterrar las semillas, constituyen rasgos pertinentes de la descripción de la agricultura selvática. Ajeno a estas prácticas, el uso del arado recién se generalizará en la región durante la primera mitad del siglo XX. Introducido por los jesuitas en el siglo XVII, nunca fue adoptado completamente por los guaraníes (Sarreal 2013), y su presencia marginal en la agricultura vernácula se consideró un obstáculo (Yssouribehere 1904).

En síntesis, el consorciamiento vegetal constituye una práctica conocida en la región, que, sin embargo, sólo será explicitada como técnica en conexión con el paisaje monoespecie de la plantation. La cubierta verde y la agroforestería, impulsadas por la agroecología en Misiones, tuvieron origen en las dificultades suscitadas por las plantaciones tropicales.

\section{Producir la naturaleza: de la intimidad vegetal al artefacto técnico}

El deterioro de la tierra frenó la escalabilidad de las grandes plantaciones en las zonas calientes del globo. En fricción con la simplificación ecológica y la fabricación de nonsoels [elementos no sociológicos] (Tsing 2019 186), la sociabilidad vegetal se convirtió en el modo de producir naturaleza. Humanos y plantas removidos de sus paisajes y proyectados con fines de alienación y control [nonsoels], reivindicaron el enmarañamiento mediante la creación de agroecosistemas que reproducen la forma y la función de la selva.

Las dificultades suscitadas en las plantaciones de caña de azúcar, café, bananas y caucho impusieron la cubierta verde en reemplazo de las carpiciones. Esta técnica, señala Bertoni, constituye la reforma moderna más importante de la agricultura tropical. Consiste "en cubrir en una plantación todos los espacios vacíos - entre líneos o entre plantas - mediante una planta baja, muy frondosa y de rápido crecimiento (...) se prefieren las Leguminosas” (Bertoni 1927, 415). La práctica reaviva la colaboración entre plantas silvestres y cultivadas, ya que si bien la cubierta se siembra puede estar constituida también por hierbas espontáneas, frecuentemente medicinales, entre las que Bertoni menciona la kapuerava o hierba de santa lucía, y el motojobo o kaité mirí, semejante al camapu citado por Levi-Strauss.

La preferencia por las leguminosas se evidencia en el listado de especies para cubierta verde elaborado por Bertoni, incluyendo las distintas variedades de poroto. Uno de ellos, "el Kumandá guasú (Canavalia ensiformis) es conocido de los paraguayos; recomendable para los terrenos pobres y secos y los faldeos pedregosos" (Bertoni 1927, 223). Otro es el Taguana (Phaceolus vulgaris), patentado como Tawana Bertoni, ${ }^{9}$ es descripto como "el frijol gigante de los guaraníes (...) Tiene un largo enrame que llega á 15 ó 20 metros de modo que en el bosque sube a la copa de los árboles elevados” (Bertoni 1927, 326). La más fertilizante de las cubiertas verde es el Kumanda ingá: "de 7 a 9 metros de largo, frondosísima” (Bertoni 1927, 227). Entre las variedades enanas, rescata tres, una de las cuales es el famoso Cow Pea, denominado Caupí en Argentina, Feijão de Vacca en Brasil, cultivado como
9 Con esta denominación fue catalogado en el Inventory of seeds and plants imported, Washington Governement printing office, 1919. 
cubierta o abono verde, pero de grano comestible (Bertoni 1927, 227).

Bajo el lema "no hay agricultura moderna sin leguminosas", contempla también la fertilización llevada a cabo por las herbáceas bajas, como la mucuna y las vicias o veza, "especialmente la velluda o villosa, que es planta de invierno y da una cubierta que es un prado artificial" (Bertoni 1927, 224). Con respecto a las vicias, en la década de 1940 un colono suizo recomendaba la siembra de vicia villosa en los yerbales de Misiones, remontando el origen de esta práctica a un descubrimiento casual (Gallero 2014, 254).

Cuatro de las variedades enunciadas por Bertoni, componen el repertorio técnico de la agroecología en la región. Así, el cultivo intercalado de Canavalia ensiformis, denominado poroto sable, se impuso a partir de 1998, en el manejo orgánico de la yerba mate, principal rubro de la agricultura comercial de Misiones. La sociabilidad entre el poroto y el maíz retorna como una técnica de fertilización recomendada por el Catálogo de tecnologías para pequeños productores agropecuarios (2007), del gobierno argentino. Sembrados en forma conjunta, mezclando las semillas de maíz y poroto caupí en el tacho de la sembradora es una práctica en uso entre pequeños productores de la provincia de Chaco.

Entre los pequeños agricultores del frente pionero con los que llevo a cabo mi trabajo de campo, la técnica de la cubierta verde fue promovida por las empresas tabacaleras, a través del cultivo de avena con el fin de reutilizar para la siembra de maíz la tierra en la que fuera cultivado el tabaco. Uno de estos productores me refería: "Los que ponen mucuna o poroto sable son los que no usan el mismo rozado del tabaco para el maíz, los demás tienen que poner avena” (agricultor, Depto. San Pedro 2007).

La cooperativa semillera que nuclea a estos agricultores comercializa semillas de cubierta verde y de maíz (Schiavoni 2018). El técnico a cargo relata: "En 1998 la cubierta verde era algo desconocido, sólo la avena que daban las tabacaleras. Trajimos catorce variedades para probar y las que quedaron fueron mucuna y poroto sable. El poroto caupí es más costumbre paraguaya" (técnico-agricultor, Depto. San Pedro 2014).

La interacción vegetal escapa al accionar de la cooperativa, ya que, si bien la organización comercializa semillas de leguminosas con destino a cubierta verde y también de maíz, no contempla su combinación. Es decir, la sociabilidad vegetal, aunque conocida, se halla débilmente enunciada y no es considerada un artefacto de mediación con la naturaleza.

La agroforestería, de reciente incorporación al repertorio técnico de la agroecología regional, complejiza el consorciamiento vegetal, incluyendo múltiples variedades (alrededor de treinta) e incorpora la regulación de la luz y el calor, re-creando la dinámica de la selva, mediante la sucesión de funciones de los distintos estratos. No se trata meramente de imitar la selva, sino de dirigir hacia ella una mirada orientada por un proyecto técnico.

En efecto, la técnica en tanto operación supone la conversión de una estructura en otra. La tecnicidad es una relación de mediación mutua, de diálogo, entre los humanos y la materia que crea un dominio transindividual, metaestable y trans- 
ductivo (Simondon 2014). Considerado en términos de espontaneidad, el trabajo conjunto con las plantas no constituye una técnica, mientras que esta atribución resulta clara con respecto a los Ogm (organismos genéticamente modificados), vivientes construidos, en cierto sentido abstractos, emanados de la división entre diseño y ejecución (Micoud 2002).

En el tipo de agricultura que estamos analizando, la relativa indiferenciación entre diseño y ejecución sitúa el diálogo técnico en el marco del modelo del artesano, propio de la antropología de la técnica, y cuyas limitaciones teóricas subrayan Vaccari y Parente (2017).

Como hija de la recolección (Barrau 1967), la horticultura selvática participa, junto con la caza, de "formas de destreza, de 'copiado' atento en el mundo" (Ingold 2012, 34), en las que la agencia no está localizada exclusivamente en la mente humana, sino en la configuración emergente de la interacción con los demás vivientes.

$\mathrm{Al}$ incorporar las acciones transformativas de los no humanos, este formato de producción re-materializa la noción de trabajo, entendiéndolo en términos de sintonización con el ambiente, apelando a los parecidos y al contexto espacio-temporal compartido.

Tal como afirma Simondon (2014) en su crítica al modelo hilemórfico, la materia no es inerte y comporta formas implícitas con poder de modulación. La operación técnica utiliza dichas singularidades como información, de modo que no es una forma abstracta la que se impone sobre la materia, sino que ella limita y estabiliza algo existente. De esta manera, las técnicas de fertilización vegetal mediante cubierta verde y agroforestería explotan una mutualidad previa y emanan de una relación con lo viviente orientada por un proyecto técnico.

\section{Modular la naturaleza: mapas y analogías}

Así como los indígenas australianos unen itinerarios nómades y viajes en sueños, componiendo «un entramado de recorridos» (Deleuze 1996, 100), la agroeco-lógica, al modular las afinidades y conexiones de los sistemas vivientes, dibuja el mapa de la agricultura más que humana, yuxtaponiendo la actividad artefactual al proceder de las plantas. El desplazamiento con respecto a la concepción habitual de agroecología consiste en colocar el énfasis en la emergencia de una estructura transindividual humano-vegetal. Es decir, los humanos no están afuera, imitando a la naturaleza, sino que forman junto con las plantas un artefacto mixto.

En la crítica que realizan al formato de constitución inmediata y situada de la técnica por parte de la antropología - concentrada en la anatomía temporal del acto -, Vaccari y Parente (2017) señalan el olvido de los aspectos normativos y teleológicos del proceso que explican la permanencia y continuidad de linajes técnicos, así como el hecho que las culturas humanas seleccionan y reproducen tipos de artefactos. La habilidad, agregan, responde a esquemas previamente adquiridos que dan forma a la estructura de esa acción. Para incorporar estas causas, que denominan distales o trascendentes, proponen relativizar la noción de representación, señalando que no siempre actúa como un programa, ejemplificando 
su propuesta con los nuevos aportes sobre la intencionalidad.

Creo, sin embargo, que es posible sobrepasar la inmediatez y dar cuenta de la continuidad técnica, sin apelar a factores trascendentes. Engendrada en el diálogo entre los humanos y la materia, la técnica constituye una estructura mixta cuyo dinamismo no es explicable por procesos que ocurrirían sólo en uno de sus componentes (la mente de los humanos). La continuidad, a su vez, emana del carácter no saturado de los objetos técnicos, cuya integración creciente les proporciona cierta autonomía, evidenciada en la capacidad de engendrar familias y estirpes.

En este sentido, el lenguaje de la modulación, propuesto por Simondon (2014), según el cual la adquisición de forma se efectúa a través de un moldeado continuo, ofrece la ventaja de no suprimir la dependencia con respecto a la materia de la tecnicidad humana. La modulación introduce una heterogeneidad entre el componente y lo compuesto, pero se trata de un código que conserva una vinculación continua con aquello que codifica.

En el caso que nos ocupa, consiste en cultivar retomando operaciones de la naturaleza. El hilo conductor es aquí la analogía, cuya versión simple como transporte de similitud, caracteriza el proceder premoderno, que imita la naturaleza, siguiendo contigüidades y semejanzas ostensibles. La analogía, insiste Simondon (2014), no es similitud de propiedades sino de relaciones. El acto analógico consiste en transportar una operación, aprendida y probada en una estructura, a otra estructura. Y agrega: “el método analógico es válido si refiere a un mundo en el que los seres son definidos por sus operaciones y no por sus estructuras, por lo que hacen y no por lo que son" (Simondon 2014, 476). La agroeco-lógica, entonces, no es imitar la naturaleza, sino modular su proceder, dialogando con las formas implícitas y operando sobre el espacio (proximidad, distanciamiento), y el tiempo (sucesión y escalonamiento para regular la luz y el calor).

Las técnicas resultantes deben más a la experiencia del recorrido que a un diseño de la mente, ya que la lógica técnica emerge en yuxtaposición con asociaciones observadas.

La noción de transecto ${ }^{10}$, mencionada a propósito de Coupaye (2015), y sugerida también por Hartigan (2017) en combinación con la descripción diluida (thin description), ${ }^{11}$ propia de la etnografía multiespecie, constituyen instrumentos de modulación. Se trata de inhibir el impulso clasificatorio y cultivar la imaginación sensorial, mediante las artes de atentividad. ${ }^{12}$

Los ambientes agrarios equivalentes a costuras - los límites entre los campos y las selvas y los márgenes de las zonas de cultivo- son espacios propicios para el compañerismo (Tsing 2015).

Esta perspectiva presta un nuevo interés a fenómenos asociativos, tales como la micorriza o relación simbiótica entre un hongo y las raíces de una planta, que si bien se conocía desde fines del siglo XIX se dejó de lado porque escapaba al reduccionismo químico (Cohen, 2017). Esta noción, como la de simbiosis y la de rizosfera ${ }^{13}$ ofrecen indicios de la metaestabilidad y el carácter no saturado de lo viviente, ejemplificado magistralmente en las historias de hongos relatadas por Tsing (2015). La analogía simple, en la que la similitud es productora, da paso a
10 El transecto es una técnica de observación y registro de datos. En zonas en las que existe una transición de flora y fauna, o de parámetros ambientales, se hace un estudio detallado a lo largo de una línea (real o imaginaria) que cruza a través de la misma.

11 El enfoque multiespecie quita exclusividad a lo humano, tomando como eje las relaciones entre especies, recurriendo a un formato descriptivo que distribuye en una red el trabajo interpretativo.

12 Consisten en conocer al otro en su particularidad y aprender cómo se podría responder mejor al otro , para cultivar mundos de florecimiento mutuo (van Dooren et al. 2016).

13 Parte del suelo inmediata a las raíces e influenciada por estas. 
una similitud producida, que modula el proceder de la naturaleza.

\section{Conclusiones}

He desarrollado aquí el problema de la conexión entre lo técnico y lo viviente, a propósito de la horticultura selvática y sus devenires agroecológicos. Enunciado como una disyunción, el título plantea una alternativa entre la agroecología y una agricultura más que humana, que legitime el aporte de los vegetales al proceso de domesticación. Abrevando en la literatura antropológica sobre la técnica y las plantas cultivadas, detecté vetas que me mostraron la agricultura como una actividad mixta, fruto de la acción conjunta de humanos y vegetales.

Las observaciones de Simondon relativas a la consideración del objeto natural como el polo hacia el cual tiende la concretización técnica, me permitió avanzar en la modulación del diálogo de los humanos con la materia vegetal, dando cuerpo a una agroecología despojada de su carácter antropocéntrico. A lo largo de la argumentación, busqué transformar la analogía simple de los premodernos en un lenguaje analógico, que, aunque injerta un código en la relación con la naturaleza, lo hace mediante un ajuste continuo con respecto a la materia que codifica.

Este paralelismo entre lo técnico y lo viviente puso en evidencia una cierta autonomía de la estructura engendrada, que sería erróneo asimilar a la supresión del componente humano, ya que lo propio de la técnica es el diálogo entre el hombre y la materia. Al incluir las acciones transformativas de los no humanos, la agricultura más que humana valoriza el trabajo como sintonización continúa con el ambiente, de manera que lo artefactual radica en la posibilidad de co-coordinación con los demás vivientes, calificando los objetos - alimentos y medicinas- en términos del proceder conjunto de los humanos y las plantas.

Recebido em 01/03/2021

Aprovado para publicação em 19/10/2021 pelo editor Alberto Fidalgo Castro 


\section{Referências}

Agustín, Miguel. 1722. Libro de los secretos de agricultura, casa de campo, y pastoril. Alicante: Biblioteca Virtual Miguel de Cervantes.

Barrau, Jacques. 1967.“De l'homme cueilleur a l'homme cultivateur: l'exemple océanien". Cahiers d'histoire mondial, X(2): 275-92.

Barrau, Jacques. 1975. “Ecologie”. In Elements d'etnologie 2. Six approaches, ed. Robert Cresswell, 7-43. Paris: Armand Collin.

Bemakhlouf, Ali. 1999. "Analogie". In Dictionnaire d'histoire et philosophie des science, dir. Dominique Lecourt, 32-6. Dictionnaire d'histoire et philosophie des science. Paris: PUF.

Bertoni, Moisés. 1927. Agenda y mentor agrícola: guía del agricultor y colono. Puerto Bertoni: Ediciones Ex Sylvis.

Bertoni, Moisés. 1940. Diccionario Botánico Latino-Guaraní. Asunción: Editorial Guaraní.

Besson, Yvan. 2009. “Une histoire d'exigences: philosophie et agrobiologie. L'actualité de la pensée des fondateurs de l'agriculture biologique pour son développement contemporain". Innovations Agronomiques 4: 329-62.

Boidin, Capucine. 2005. “Jopara: una vertiente sol y sombra del mestizaje”. HALshsMunster: 303-31.

Cadogan, Leon. 2011. Diccionario Mbya-guarani-Castellano. Asunción: CEADUC.

Catálogo de Tecnologías Para Pequeños Productores Agropecuarios. 2007. Buenos Aires: Secretaría de Agricultura, Ganadería, Pesca y Alimentos de la Nación Argentina.

Coccia, Emanuele. 2017. La vida de las plantas. Una metafísica de la mixtura. Buenos Aires: Miño y Dávila.

Cohen, Aurélien Gabriel. 2017. "Des lois agronomiques à l'enquête agroécologique. Esquisse d'une épistémologie de la variation dans les agroécosystèmes". Tracés. Revue de sciences humaines 33: 51-72.

Conklin, Harold. 1954. "An ethnoecological approach to shifting agriculture". Transactions of the New York Academy of Sciences 17(2): 133-42.

Coupaye, Ludovic. 2015. «Chaîne opératoire, transects et théories: quelques réflexions et suggestions sur le parcours d'une méthode classique ». In L 'homme tout simplement, ed. Philippe Soulier, e André Leroi-Gourhan, 69-84. Paris: De Boccard.

Cresswell, Robert. 1983. "Evolution humaine: techniques et cultures". In Éléments d'ethnologie. 1-Huit terrains, ed. Robert Cresswell, 60-85. Paris: Armand Colin.

Deleuze, Gilles. 1996. Crítica y clínica. Barcelona: Anagrama.

Descola, Philippe. 1988. La selva culta. Simbolismo y praxis en la ecología de los Achuar. Quito: Coedición Instituto Francés de Estudios Andinos y Ediciones Abyla-Yala.

Descola, Philippe. 2012. Más allá de naturaleza y cultura. Buenos Aires: Amorrortu.

Emperaire, Laure, e Manuela Carneiro da Cunha. 2010. "Transformar o espaço”. In Dossiê de registro do Sistema Agrícola Tradicional do Rio Negro, org. Laure Emperaire, 51-72. Brasília: ACIMRN; IPHAN; IRD; Unicamp-CNPQ.

Felipim, Adriana. 2001. "O sistema agrícola guarani mbyá e seus cultivares de milho: um estudo de caso na aldeia guarani da llha do Cardoso, município de Cananéia, São Paulo". Dissertação de mestrado, Universidade de São Paulo. 
Ferret, Carole. 2012. Vers une anthropologie de l'action André-Georges Haudricourt et l'efficacité technique. L'Homme, 202: 113-139.

Fleck, Eliane, e Mariana Alliatti. 2017. “Sobre 'Los hijos del Paraguay’ y las ‘Personas naturales inteligentes': un análisis de los relatos sobre saberes y prácticas tradicionales indígenas en el Paraguay Natural Ilustrado, de José Sánche Labrador S. J. (1771-1776)". Memoria Americana, 25(2): 29-46.

Fleck, Eliane, Mariana Alliatti, e Maico Biehl. 2016. "En orden a sus virtudes y facultades medicinales: un estudio sobre o Paraguay Natural Ilustrado de José Sánchez Labrador S. J.”. Corpus, 6(2). https://doi.org/10.4000/corpusarchivos.1614.

Fleck, Eliane. 2017. "A medicina da conversão: apropriação e circulação de saberes e práticas de cura (Província Jesuítica do Paraguay, século XVIII”. Revista de Estudios Marítimos y Sociales: $34-80$.

Foucault, Michel. 1966. Les mots et les choses. Paris: Gallimard.

Gallero, Cecilia, ed. 2014. Querida Misiones, hermosa! Querida Misiones, hermosa!: a través de las memorias de Alberto Roth. Buenos Aires: Araucaria.

Geertz, Clifford. 1970. Agricultural involution: the processes of ecological change in Indonesia. Berkeley: University of California Press.

Gourou, Pierre. 1949. “Qu'est-ce que le monde tropical ?”. Annales. Economies, Sociétés, Civilisations 4(2): 140-48.

Hartigan, John. 2017. Care of the Species: Races of Corn and the Science of Plant Biodiversity. Minnesota: University of Minnesota Press.

Haudricourt, André-George, e Louis Hédin. 1943. L'homme et les plantes cultivées. Paris: Gallimard.

Haudricourt, André-George. 1962. "Domestication des animaux, culture des plantes et traitement d'autrui”. L'Homme 2(1): 40-50.

Ingold, Tim. 2012. Ambientes para la vida. Conversaciones sobre humanidad, conocimiento y antropología. Montevideo: Trilce.

Ingold, Tim. 2013. "La textilité de la fabrication”. In: Marcher avec les dragons, Tim Ingold, 221-38. Bruselas: Zones Sensibles.

Keller, Héctor. 2007. "Notas sobre medicina y magia entre los guaraníes de Misiones: un enfoque etnobotánico”. Suplemento antropológico: 345-83.

Klein, Ursula. 2005. "Shifting ontologies, changing classifications: plant materials from 1700 to 1830". Studies in History and Philosophy of Science, 36: 261-329.

Lagasca, Mariano. 1826. Observaciones sobre la familia natural de las plantas aparasoladas (umbelliferae). Londres: Imprenta Macinstosh.

Lemonnier, Pierre. 2013. "De l'immatériel dans le matériel ... et réciproquement ! Techniques et communication non verbal". Journal de la Société des Océanistes, 136-137: $15-26$.

Leroi-Gourhan, André. 1964. Le geste et la parole. Technique et Langage. Paris: Albin Michel.

Leroi-Gourhan, André. 1973. Milieu et techniques. Paris: Albin Michel.

Lévi-Strauss, Claude. 1962. La pensée sauvage. Paris: Plon.

Lévi-Strauss, Claude. 1971. De la miel a las cenizas. México: FCE.

Mafferra, Luis. 2019. “El libro de los secretos de la agricultura del Fray Agustín y las 
relaciones humano-plantas durante la modernidad temprana". Revista del Museo de Antropología, 12(3): 85-96.

Martin, Carmen, e José Luis Valverde. 1995. La farmacia en la América colonial: el arte de preparar medicamentos. Granada: Servicio de Publicaciones de la Universidad de Granada.

Micoud, André. 2002. "Les OGM: des objets vivants construits?". In Simondon: une pensée opérative?, coord. Jacques Roux, 189-203, Simondon: une pensée opérative?. Sain Etienne: Publications de l'Université de Saint-Etienne.

Montenegro, Pedro. 2007. Materia Medica Misionera. Posadas: Editorial Universitaria de Misiones.

Montoya, Antonio Ruiz de. 1876 [1640]. Bocabulario de la lengua guaraní. Leipzig: Edición de J. Platzmann.

Montoya, Antonio Ruiz de. 2011[1639]. Tesoro de la lengua guaraní. Asunción: Cepag, Edición de F. Grünberg y B. Melià.

Müller, Franz. 1989. Etnografía de los Guaraní del Alto Paraná. Rosario: Escuela de Artes Gráficas del Colegio Salesiano San José.

Müller, Germán. 1995. Memorias de Heiner Müller: pionero de Montecarlo. Posadas: Editorial Universitaria de Misiones.

Otazu Melgarejo, Angélica. 2014. "Contribución a la medicina natural: Pohã Ñana, un Manuscrito inédito en Guaraní (Paraguay, S. XVIII)”. Corpus 4(2).

Racine, Luc. 1989. "Du modèle analogique dans l'analyse des représentations magico-religieuses". L'Homme, 29(109): 5-25.

Schaden, Egon. 1974. Aspectos fundamentais da cultura guarani. São Paulo: Editora da Universidade de São Paulo.

Schiavoni, Gabriela. 1995. Colonos y ocupantes: parentesco, reciprocidad diferenciación social en la frontera agraria de Misiones. Posadas: Editorial Universitaria de Misiones.

Schiavoni, Gabriela. 2018. "De las familias criollas a las lignées purificadas. Analogías entre vegetales y humanos". Anuário Antropológico, 43(2): 309-34.

Schiavoni, Gabriela. 2020. "Concordancias ontológicas e hibridaciones técnicas Los alimentos agroecológicos en Misiones". In El mundo rural y sus técnicas, org. Ana Padawer, 179-213. Ciudad Autónoma de Buenos Aires: Editorial de la Facultad de Filosofía y Letras, Universidad de Buenos Aires.

Sigaut, François. 1982. "Techniques et société chez les cultivateurs de tubercules : quelques réflexions critiques". Journal d'agriculture traditionnelle et de botanique appliquée 29e , 3(4): 355-64.

Simondon, Gilbert. 2014. La individuación a la luz de las nociones de forma y de información. Buenos Aires: Cactus.

Souza, José. 1987. "Uma introdução ao sistema técnico-econômico guarani”. Dissertação de mestrado, Universidade Federal do Rio Grande do Sul.

Tsing, Anna. 2019. Viver nas ruínas. Paisagens multiespécies no Antropoceno. Brasília: IEB Mil Folhas.

Vaccari, Andrés, e Diego Parente. 2017. "Materialidad e intencionalidad. Algunas dificultades con la teoría de la agencia material y el enfoque ecológico". Estudios Filosóficos: 152-78.

van der Ploeg, Jan Douwe. 2000. “Sistemas de conocimiento, metáfora y campo de 
interacción: el caso del cultivo de la patata en el altiplano peruano". In Antropología del desarrollo. Teorías y estudios etnográficos en América Latina, comp. Andreu Viola, 361-83. Barcelona: Paidós.

van Dooren, Thom, Eben Kirskey, e Ursula Münster. 2016. "Estudos multiespécies: cultivando artes de atentividade". ClimaCom Cultura Científica: 39-66.

Van Dooren, Thom. 2012. "Wild Seed, Domesticated Seed. Companion species and the emergence of agriculture”. Philosophy, Activism, Nature, 9: 22-28.

Willis, Rick. 2007. The history of Allelopathy. Dordrecht: Spinger.

Woortmann, Ellen. 1997. O trabalho da terra: a lógica e a simbólica da lavoura camponesa. Brasília: Editora da Universidade de Brasília.

Yssouribehere, Pedro. 1904. Investigación agrícola en el territorio de Misiones. Anales del Ministerio de Agricultura 1, 9. Buenos Aires: Compañía Sud-Americana de Billetes de Banco. 


\section{Mapa de la región de Misiones (Argentina)}

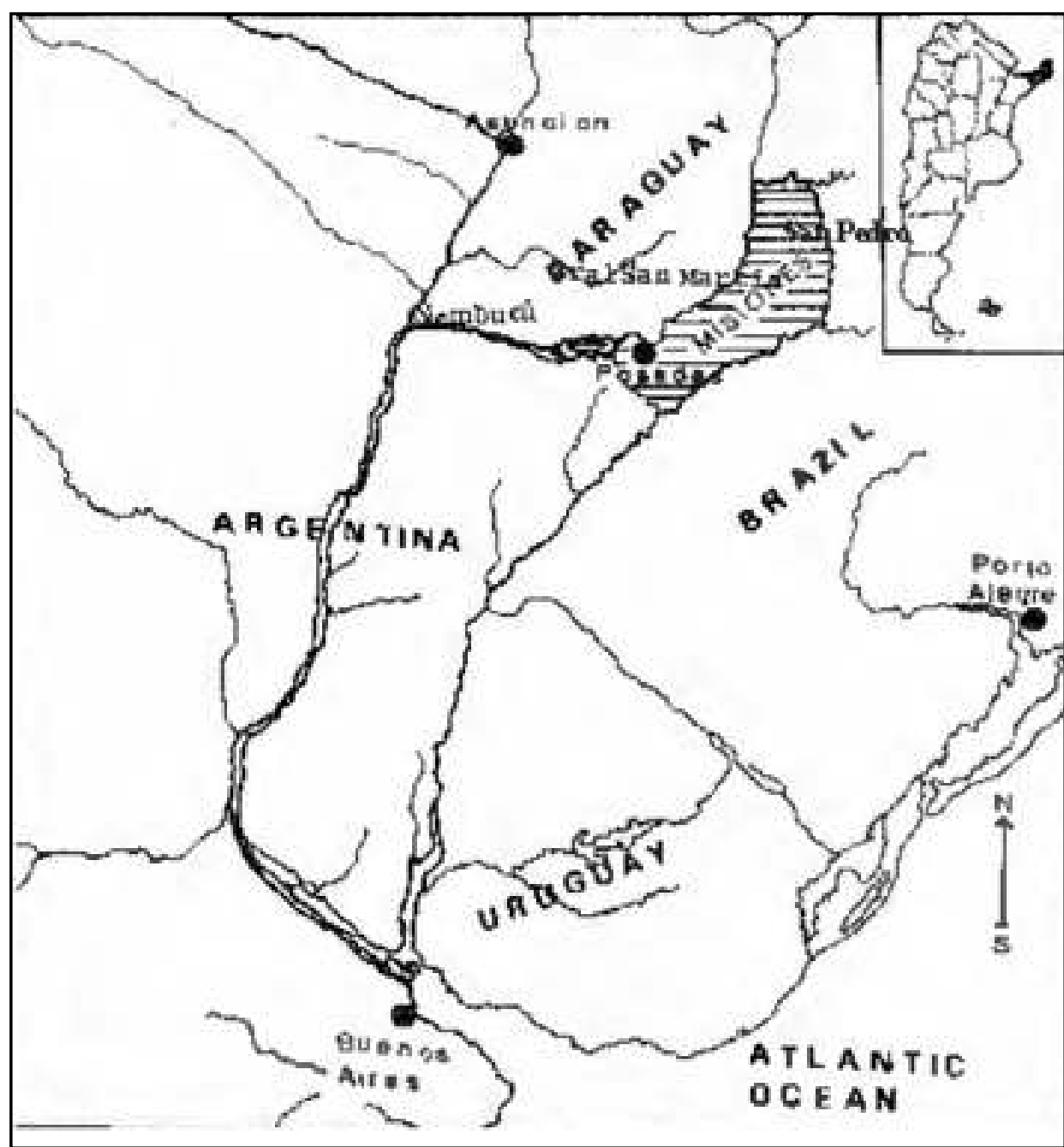

Fuente: Adaptado de

Bartolomé, L. 1989. In The Human Ecology of Tropical Land Settlement in Latin America, eds. Schumann y Partrid. Westview Press, Boulder-San Francisco \& London. 